\title{
Optimal Design of Double Folded Stub Microstrip Filter by Neural Network Modelling and Particle Swarm Optimization
}

\author{
Amir Banookh, S. Masoud Barakati \\ Faculty of Electrical and Computer Engineering, The University of Sistan and Baluchestan, Iran \\ Email:amirbanookh@ece.usb.ac.ir,smbaraka@ece.usb.ac.ir
}

\begin{abstract}
Optimization of design parameters based on electromagnetic simulation of microwave circuits is a timeconsuming and iterative procedure. To provide a fast and accurate frequency response for a given case study, this paper employs a neural network modelling approach. First, one of the case study's outputs, i.e., scattering parameter $\left(\left|S_{2 I}\right|\right)$ in $\mathrm{dB}$, is predicted using a neural network model. Then the particle swarm optimization is employed to optimize the design parameters. The proposed method in designing the filter compares with two others methods for a case study. The simulation results show the capability of the proposed method in designing an optimized filter in a proper time.
\end{abstract}

Index Terms - neural networks, Microwave structures, particle swarm optimization, modelling.

\section{INTRODUCTION}

Neural networks are information processing systems inspired by the human brain's ability to learn from observations [1]. Neural networks are efficient alternatives to conventional methods, such as numerical modelling methods which could be computationally expensive, or analytical methods which could be difficult to obtain, or empirical models whose range and accuracy could be limited. Due to their ability and adaptability to learn, generalizability, fast real-time operation, and ease of implementation, they have been used in microwave design problems, RF, and microwave computeraided design problems [2].

In microwave applications, a neural network can train to mimic the electrical behavior of the circuit. Then, it can be used in high-level simulation and design, providing a fast response to the learned task [3], [4]. Moreover, neural networks can be employed along with evolutionary algorithms such as genetic algorithm [5] and particle swarm optimization [6] to optimize the system under study. In this application, a neuarl network can use with an electromagnetic packages, such as HFSS, CST, ADS, to optimize the design.

In this paper, to accelerate the frequency sweep required in the full-wave simulators, for calculating the $S$-parameters of a double folded stub microstrip filter, an artificial neural network is employed. The neural network is trained, by achieved patterns from an electromagnetic package, to approximate the $S$-parameters in the region of interest. The proposed model has capability to perform the frequency 
sweep fast and accurate, in comparison with the available electromagnetic packages. To optimize the designed model the particle swarm optimization method is used.

The neural network structure is explained in the next section. Section 3 is dedicated to describe the of particle swarm optimization. The case study is introduced in section 4. To verify the proposed model, simulation results are illustrated in section 5. The last section is conclusions.

\section{THE STRUCTURE OF NEURAL NETWORK}

In this section, the structure of neural networks and its ability to model the behavior of RF and microwave components are described.

The MLP is a commonly used neural network structure, in which the neurons are grouped into layers. The MLP contains input, hidden, and output layers. Each layer consists of some nodes and fully connected to the next one. Node (or neuron) is a processing element with a nonlinear activation function. The activation functions are applied to the weighted sum of the inputs of a neuron to produce the output. Fig. 1 shows the structure of MLP neural network; consist of one input layer, three hidden layers, and one output layer.

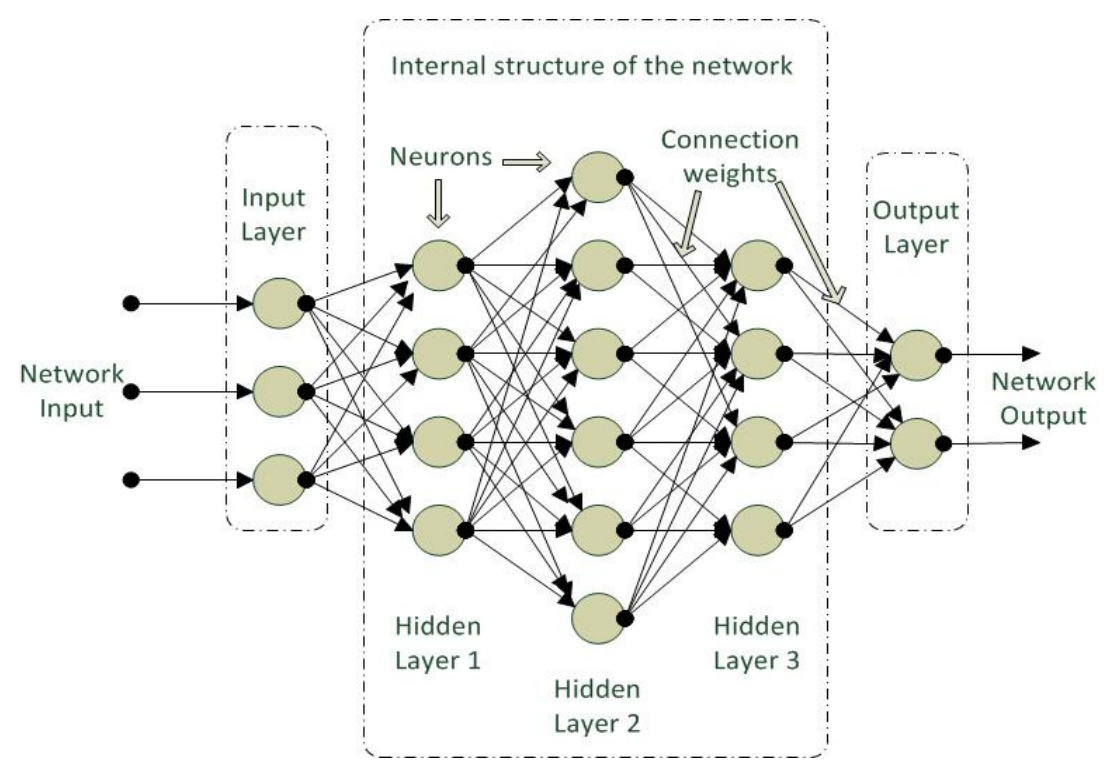

Fig. 1. Structure of the MLP artificial neural networ

Input neurons receive stimulus from outside the network, while the output of output neurons are externally used. Hidden neurons receive stimulus from some neurons and their outputs are stimulus for some other neurons. Different neural networks can be constructed by using different types of neurons and by connecting them in different configurations [8].

The weights are the most important factor in determining the MLP function. Training is the act of presenting the network with some sample data and modifying the weights to better approximate the desired function. The weighting parameters, which are real numbers, are initialized before training the MLP. During training, they are updated iteratively in a systematic manner. Typically many iterations (or epoch) are required to train MLP. To train MLP a learning method, such as a supervised 
backpropagation learning technique can be used. The basic backpropagation algorithm is based on minimizing the error of the network using the derivatives of the error function. Most common measure of error is the mean square error: $\mathrm{E}=(\operatorname{target}-\text { output })^{2}$. Partial derivatives of the error with respect to the weights:

Output Neurons: $\partial \mathrm{E} / \partial \mathrm{w}_{\mathrm{ji}}=-$ output $_{\mathrm{i}} \delta_{\mathrm{j}}$,

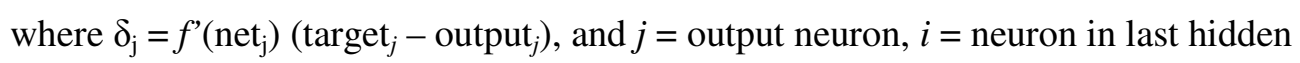

Hidden Neurons: $\partial \mathrm{E} / \partial \mathrm{w}_{\mathrm{ji}}=-$ output $_{\mathrm{i}} \delta_{j}$,

where $\delta_{\mathrm{j}}=f^{\prime}\left(\right.$ net $\left._{\mathrm{j}}\right) \Sigma\left(\delta_{k} w_{k j}\right), j=$ hidden neuron, $i=$ neuron in previous layer $k=$ neuron in next layer.

Calculation of the derivatives flows backwards through the hidden and input layers of the network. These derivatives point in the direction of the maximum increase of the error function. A small step (learning rate) in the opposite direction will result in the maximum decrease of the (local) error function:

$w_{\text {new }}=w_{\text {old }}-\alpha \partial E / \partial w_{\text {old }}$,

where $\alpha$ is the learning rate.

Once the neural network training is completed, the weighting parameters remain fixed throughout the usage of the neural network as a model.

The case-study is modeled by a multilayer perceptron (MLP) neural network simulated in a MATLAB environment [7].

\section{PARTICLE SWARM OPTIMIZATION}

In this section, a brief description of particle swarm optimization (PSO) is presented. PSO is a stochastic optimization technique [9]-[16], that was developed in 1995 by Eberhart and Kennedy. It is a global optimization algorithm, which can effectively be used to solve multidimensional optimization problems [17]. PSO attempts to simulate the swarming behavior of birds, bees, fish, etc. It aims at increasing the probability of encountering global extremum points without performing a comprehensive search of the entire search space. PSO can easily be implemented and its performance is comparable to other stochastic optimization technique, such as genetic algorithm and simulated annealing [14].

The PSO starts with an initial population of individuals, which are named swarm of particles. Each particle in the swarm is randomly assigned an initial position and velocity. The position of the particle is an $\mathrm{N}$-dimensional vector, representing a possible set of optimization parameters. Each particle starts from its initial position, $x_{i}=\left[x_{i 1}, x_{i 2}, \ldots, x_{i n}\right]^{T}$, and its initial velocity, $v_{i}=\left[v_{i 1}, v_{i 2}, \ldots, v_{i n}\right]^{T}$. According to the individual and the swarm experience, the velocity and position of each particle are updated during the algorithm search. The goodness of a new particle position, a possible solution, is measured by evaluating a fitness function. The process of updating velocities and positions continues by the time that one of the particles in the swarm finds the position with best fitness. Since the particles will not be able to find a better one, all of them will eventually be drawn to this position. 
The main steps of PSO algorithm can be summarized as follows:

\section{A. Definition of the solution space}

In this step, the minimum and maximum values for each dimension in the N-dimensional optimization problem are specified.

\section{B. Definition of the fitness function}

The fitness function is a problem-dependent parameter which is used to evaluate and measure the goodness of a position representing a valid solution.

\section{Random initialization of the swarm positions and velocities}

The position and velocity of the particles are randomly initialized. For faster convergence, however, it is preferred that they are randomly chosen from the solution space. The number of particles is problem-dependent, and for most engineering problems a swarm size of thirty particles is good enough [18]. To complete the initialization step, the initial position of each particle is labeled as the particle's best position, Pbest. Also, the position of the particle with best fitness among the whole swarm is labeled as the global best position, Gbest. The word 'best' means highest or lowest depending on whether the problem is a minimization or maximization problem.

\section{Updating the velocity and position of a particle}

In this step, the following sub-steps are carried out for each particle

- Velocity update

The velocity of the $i^{\text {th }}$ particle in the $j^{\text {th }}$ dimension, $v_{i j}$ is updated according to (4)

$$
v_{i j}(t+1)=w v_{i j}(t)+c_{1} r_{1}\left(\text { Pbest }_{i j}-x_{i j}(t)\right)+c_{2} r_{2}\left(\text { Gbest }{ }_{j}-x_{i j}(t)\right), j=1,2, \ldots, N,
$$

where $x_{i j}$ represents the position of the $i^{\text {th }}$ particle in the $j^{\text {th }}$ dimension. The time index of the current and previous iterations are denoted by ' $t+l$ ' and ' $t$ ', respectively. $r_{l}$ and $r_{2}$ are two random numbers in range [0-1], generated by uniformly distributed random functions. The parameters $c_{1}$ and $c_{2}$ are the relative weight of the personal best position and the relative weight of the global best position, respectively. Both $c_{1}$ and $c_{2}$ are typically chosen in range [0-2] [17]. The parameter $w$, which is called the "inertial weight", is a number in the range [0-1]. It specifies the weight by which the particle's current velocity depends on its previous velocity. The references [19]-[26] proposed a method on selecting and optimizing the PSO parameters.

- Position update

The position of the $i^{t h}$ particle in the $j^{\text {th }}$ dimension is updated according to the following equation

$$
x_{i j}(t+1)=x_{i j}(t)+v_{i j}(t+1), j=1,2, \ldots, N
$$

- Fitness evaluation

First, the fitness of the updated N-dimension position in the previous step is evaluated. If the returning number is better than the fitness of the Pbest, the updated $\mathrm{N}$-dimension position is labeled as 
a new Pbest. Also, if the returning number is better than the fitness corresponding to the Gbest, the updated N-dimension position is labeled as a new Gbest.

\section{E. Checking the termination criterion}

The algorithm may be terminated if the number of iterations reaches a pre-specified maximum number of iterations or the fitness corresponding to the Gbest is close enough to a desired number. If none of these conditions is satisfied, the algorithm will return to the previous step.

\section{CASE-STUDY: DOUBLE FOLDED STUB MICROSTRIP FILTER}

The structure of Double Folded Stub Microstrip filter in High Frequency Simulation Software (HFSS) is shown in figure. 2. It is made of a perfect conductor on the top of a Duroid substrate with a relative dielectric constant of 9.9 and a height of $5 \mathrm{mil}$, and the bottom layer is made with a perfect conductor ground plane. The two design variable parameters are $S$ and $L_{2}$ and three other design parameters, $w_{1}, w_{2}$, and $L_{1}$ are fixed at 4.8, 4.8, and 93.7 mil, respectively. The diagram of Double Folded Stub Microstrip filter, that shows the design parameters, is illustrated in figure 3. To represent such input-output relationship, an artificial neural network model is trained for given design parameters within the region of interest. The neural network model is used to provide a fast estimation of $\left|S_{21}\right|$ based on given filter's geometrical parameters.

Consider $x, y$, and $w$ vectors refer to the neural network input, output, and weighting parameters, respectively. The neural network input-output relationship is given by

$$
y=f(x, w) .
$$

The neural network input is given by

$$
x=\left[s L_{2} \omega\right]^{T},
$$

where $\omega$ is a range of frequency. The sample points are generated using an electromagnetic simulator, i.e., HFSS [27]. The region of interest, in mil, is $3.6 \leq S \leq 7.6$ and $80.3 \leq L_{2} \leq 100.3$. The objective of training is to adjust neural network weighting parameters, such that the neural model output best match to the output data of HFSS simulation.

The $\left|S_{2 l}\right|$ is computed by HFSS package at range 6 to 20 frequency points with step size $0.05 \mathrm{GHz}$. 


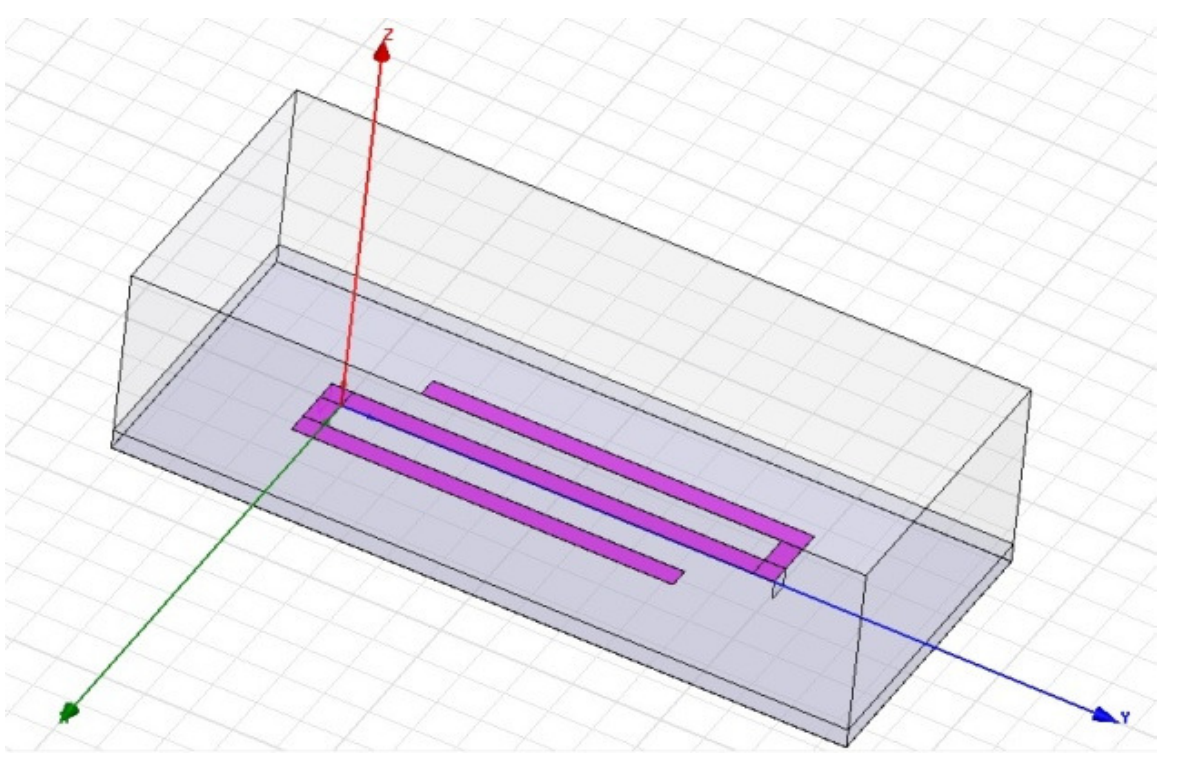

Fig. 2. Structure of Double Folded Stub Microstrip filter in HFSS software

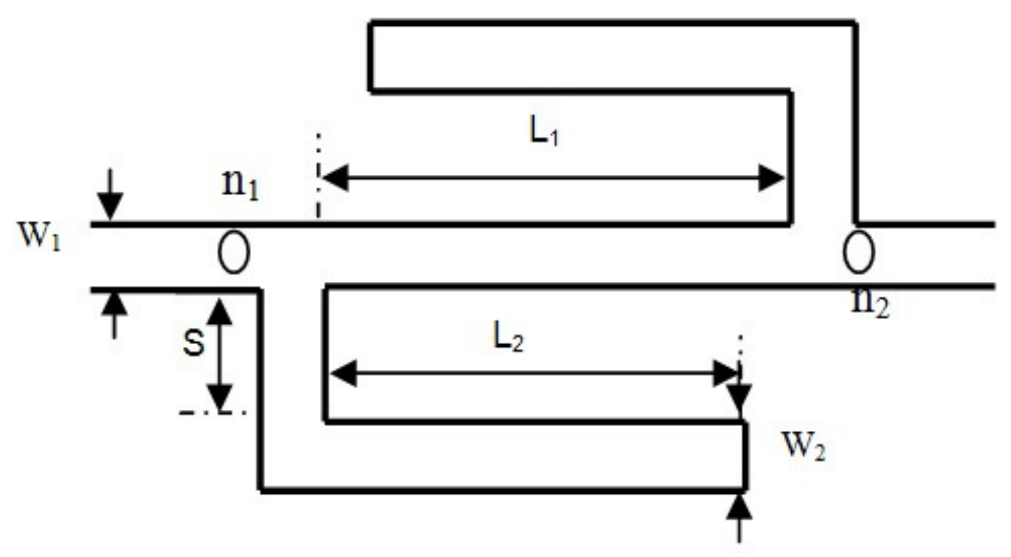

Fig. 3. Diagram of Double Folded Stub Microstrip filter

\section{SIMULATION RESULTS}

\section{A. The Neural Network training using HFSS output data}

As mentioned in previous section, an MLP neural network trained based on HFSS output pattern to approximate the $\left|S_{21}\right|$ parameters in the region of interest. The neural network model will perform the frequency sweep fast and accurate in comparison with HFSS.

The neural network consists of one input, one hidden, and one output layer. The input, hidden, and output layers contain of three neurons, 40 neurons, and one neuron, respectively. The hidden and output layer transfer functions are hyperbolic tangent sigmoid and linear functions, respectively. The 41 input vectors $x$, in form of equation (7) and in uniformly distributed sample points, are used as training patterns. The traing algorithm is performed using a bach mode and Levenberg-Marquardt method [7],[8]. During the trainnig algorithm the validation and testing of the results are very important to obtain a well-generalised MLP without overtraining [7], [28]. The trainig data will be employed to train the MLP, while the validation data will be used to check the performanceof the 
model. Proper division of the data is controlled by checking the plots of test and validation errors, at the same time [28]. If test and validation error plots do not demonstrate similar characteristics, this is an indication of poorly divided data. In this study, the input data is devided into three sets, randomly, as: $60 \%$ for traing, $20 \%$ for validation, and $20 \%$ for performance test. The learning rate, maximum number of epoch, and maximum mean square error are set to $0.1,1000,0.0001$, respectively. Figure 4 shows the training, validation, and testing errors versus number of epochs (iterations) for the MLP in the case study. The training algorithm terminates if the validation error increases (after six sequence iterations), or the limitations of maximum error/epoch are exceeded. As illustrated in Figure 4 the training algorithm is terminated in 246 epochs (because of validation error), while the training error is small enough (0.000166), and the test and validation error show similar characteristics.

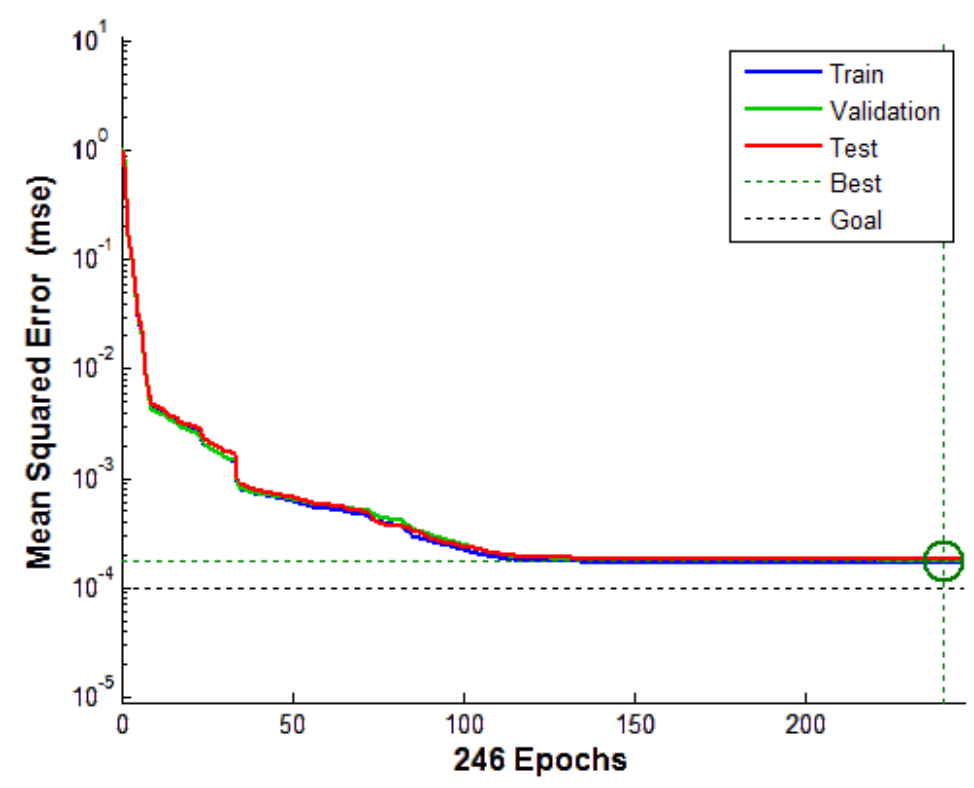

Fig. 4. The training, validation, and testing errors versus number of epochs (iterations) for the MLP

After training stage, the neural network model is evaluated based on 40 sample points.

The $\left|S_{21}\right|$ parameter of Double Folded Stub Microstrip filter is simulated by HFSS package and proposed neural network model for three different filters. The simulation results are depicted in figure 5, where two models are compared. As shown in the figure, the models responses are fairly close. Therefore, the trained neural network output can use instead of HFSS response. This replacing helps the designer to have a fast and accurate response.
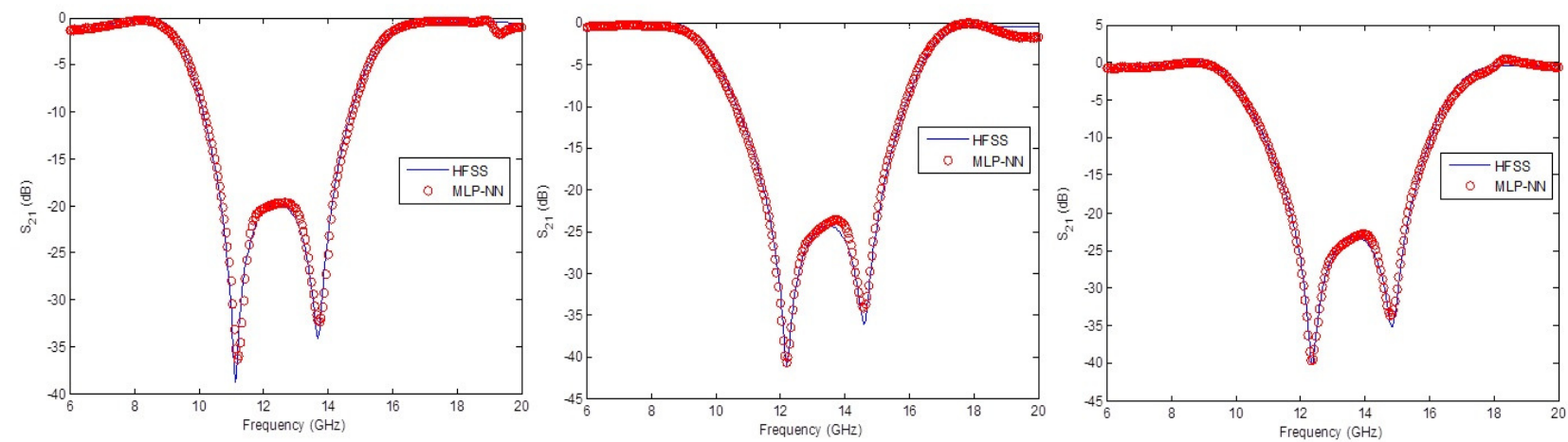
Fig. 5. $S_{21}$ frequency responses of HFSS and the Neural Network models for three different filters

\section{B. Optimization the design parameters using PSO technique}

To find the optimal values of the physical parameters, the PSO technique, introduced in section 3, is adopted. To optimize the double folded stub filter of figure 2 , the following design specifications are considered.

$$
\begin{array}{ll}
\text { I) } & S_{21} \geq-3 d B \text { for } f \leq 9.5 \mathrm{GHz} \text { and } f \geq 16.5 \mathrm{GHz} \text {, } \\
\text { II) } & S_{21} \leq-25 \mathrm{~dB} \text { for } 12 \mathrm{GHz} \leq f \leq 14 \mathrm{GHz} .
\end{array}
$$

The number of particles and total number of iterations in PSO are 80 and 100, respectively. Other PSO parameters are $c_{1}=1, c_{2}=2$, and $w=0.9$.

The optimal parameters given by PSO are: $S=7.16 \mathrm{mil}$ and $L_{2}=86.85$ mil. These optimal parameters should satisfy the design conditions I and II. For this aim, the optimal parameters are given to the HFSS software to calculate scattering parameters. Figure 6 shows $\left|S_{21}\right|$ parameter for the optimal design parameters. As shown in the figure, the conditions I and II are satisfied.

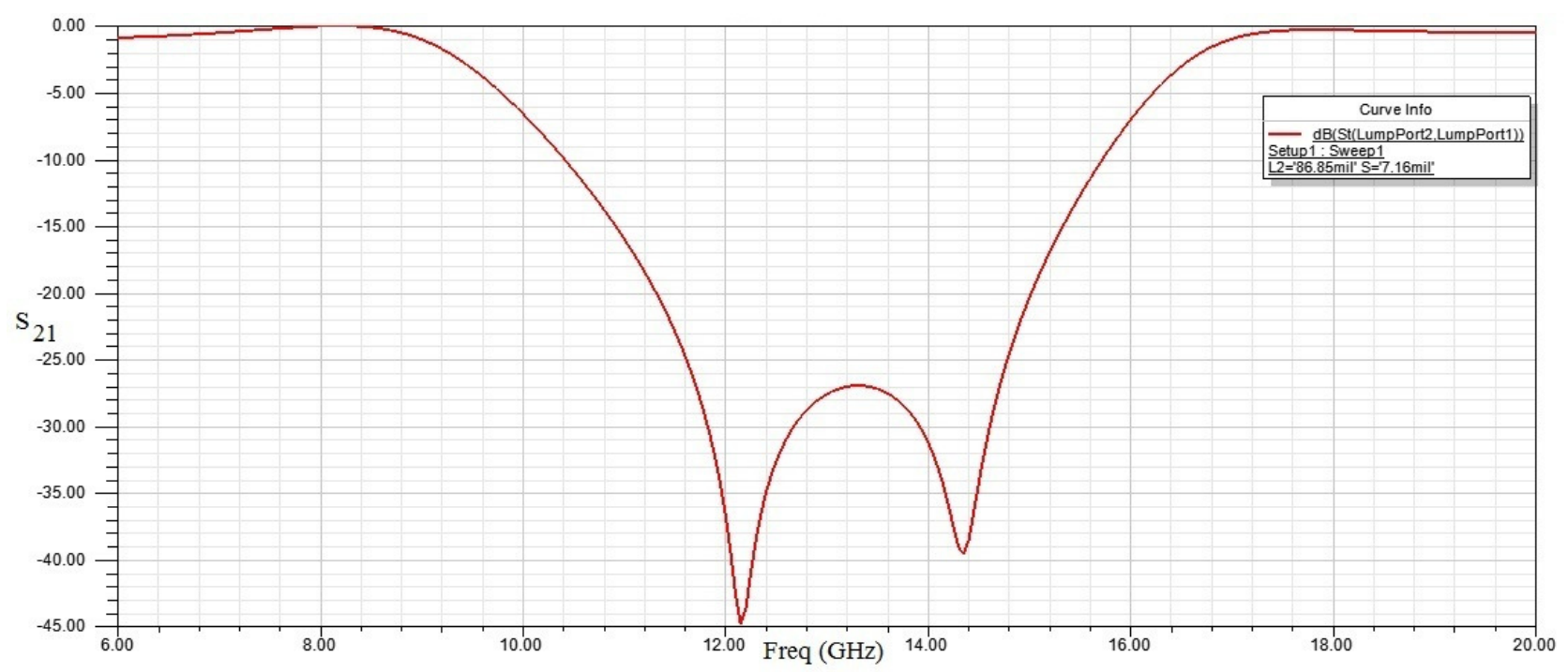

Fig. 6. Response of the Double Folded Stub Microstrip filter for optimal parameters

Table I compares three design methods for the case study, i.e., using HFSS package, using HFSS combined with PSO, and the proposed method. Substituting the trained neural network with HFSS package speeds up finding the optimal parameters of the filter. The running time of the simulated proposed method, using a machine with CPU Corei7 1.73GHz and 4G RAM, takes about 17 minutes, while using a direct PSO method in HFSS (where the number of particles and the total number of iterations in PSO are 80 and 100, and HFSS runs 8000 times) it takes about 3466 minutes, i.e., 208 times slower than the proposed method. In addition, the proposed method obtains the optimal design parameter, and the HFSS package and PSO can be linked in MATLAB, instantaneously. 
Table I. comparison the proposed method with two tools for finding the optimal parameters of the filter

\begin{tabular}{|l|c|c|c|}
\hline Design approach & $\begin{array}{l}\text { Electromagnetic } \\
\text { Package (HFSS) }\end{array}$ & HFSS-PSO & $\begin{array}{l}\text { HFSS-ANN-PSO } \\
\text { (Proposed method) }\end{array}$ \\
\hline $\begin{array}{l}\text { Design and optimization are } \\
\text { linked }\end{array}$ & - & NO & Yes \\
\hline $\begin{array}{l}\text { Running Time } \\
\text { (for the case study) }\end{array}$ & Very slow & 3466 mins & 17 mins \\
\hline
\end{tabular}

\section{Conclusion}

To optimal design of $\left|S_{21}\right|$ parameter of a Double Folded Stub Microstrip filter using an electromagnetic package, such as, HFSS, is a time consuming method. Although, the optimal parameter of the filter can be obtained by an optimization method, the HFSS package and the optimization method cannot link together easily. In this paper, an MLP neural network, trained based on the HFSS responses, is adopted for the fast and accurate calculation of $\left|S_{2 l}\right|$ parameter of the filter. The trained neural network model has capability of providing an accurate frequency response in a fraction of a second. In addition, to optimize the designed filter based on the neural network response, a particle swarm optimization (PSO) method is employed to achieve the optimal parameter. The trained MLP and PSO algorithm are interfaced in MATLAB, while for finding the optimal response using the HFSS package, an optimization algorithm should be executed off-line. Based on the simulation results for a case study, the proposed method is 208 times faster than using HFSS package. Therefore, it can conclude that the proposed method is a fast and accurse tools for designing a Double Folded Stub Microstrip filter.

\section{REFERENCES}

[1] Q. J. Zhang and K. C. Gupta, Neural Networks for RF and Microwave Design, Norwood, MA: Artech House, 2000.

[2] Q. J. Zhang and K. C. Gupta, "Neural Networks for RF and Microwave Design- From Theory to Practice,"IEEETransactions on Microwave Theory and Techniques, vol. 51, April 2003.

[3] K. C. Gupta, "Emerging trends in millimeter-wave CAD," IEEETransactions on Microwave Theory and Techniques, vol. 46, pp. 747-755, June 1998.

[4] V. K. Devabhaktuni, M. Yagoub, Y. Fang, J. Xu, and Q. J. Zhang, "Neural networks for microwave modeling: Model development issues and nonlinear modeling techniques," International Journal of RF MicrowaveComputer-Aided Engineering, vol. 11, pp. 4-21, 2001.

[5] S. N. Sivanandam and S. N. Deepa, Introduction to Genetic Algorithms, Springer, 2010.

[6] M. Clerc, Particle Swarm Optimization, John Wiley \& Sons, 2010.

[7] MATLAB, version 7.11.0.584 (R2010b), The MathWorks Inc., 2010.

[8] F. Wang, V. K. Devabhaktuni, C. Xi, and Q. J. Zhang, "Neural network structures and training algorithms for microwave applications," International Journal of RF Microwave Computer-Aided Engineering, vol. 9,pp. 216-240, 1999.

[9] R.C. Eberhart\& J. Kennedy, A new optimizer using particle swarm theory, Proc. Sixth International Symposium on Micromachine and Human Science, Nagoya, Japan, 1995, 39-43.

[10] J. Kennedy \& R.C. Eberhart, Particle swarm optimization, Proc. IEEE International Conference on Neural Networks, Piscataway, NJ, 1995, 1942-1948.

[11] J. Kennedy \& R.C. Eberhart, A discrete binary version of the particle swarm algorithm, Proc. IEEE International Conference on Systems, Man, and Cybernetics: Computational Cybernetics and Simulation, vol. 5, 1997, 4104-4108.

Brazilian Microwave and Optoelectronics Society-SBMO received 1 Febr. 2012; for review 10 Febr. 2012 ; accepted 22 June 2012 Brazilian Society of Electromagnetism-SBMag 
[12] J. Kennedy, R.C. Eberhart, \& Y. Shi, Swarm intelligence (San Francisco: Morgan Kaufmann Publishers, 2001).

[13] J. Kennedy \& R. Mendes, Population structure and particle swarm performance, on Proc. Congress Evolutionary Computation (CEC 02), vol. 2, 2002, 1671-1676.

[14] J. Kennedy \& J.M. Spears, Matching algorithms to problems: An experimental test of the particle swarm and some genetic algorithms on the multimodal problem generator, Proc. IEEE World Congress Computational Intelligence Evolutionary Computation, 1998, 78-83.

[15] S. Tavakoli and A. Banookh, Robust PI Control Design Using Particle Swarm Optimization, Journal of Computer Science and Engineering, vol 1, issue 1, p36-41, 2010

[16] M. Zeinadini, S. Tavakoli and A. Banookh, Modelling and Design of Microwave Structures Using Neural Networks and Particle Swarm Optimization, Journal of Telecommunication, vol 3, issue 1, 2010.

[17] R.C. Eberhart\& Y. Shi, Evolving artificial neural networks, Proc. Int. Conf. Neural Networks Brain, Beijing, P.R.C., 1998.

[18] J. Robinson \& Y. Rahmat-Samii, Particle swarm optimization in electromagnetics, IEEE Transactions on Antennas and Propagation, 52(2), 2004, 397-402.

[19] I.C. Trelea, The particle swarm optimization algorithm: Convergence analysis and parameter selection, Information Processing Letters, 85, 2003, 317-325.

[20] M. Clerc and J. Kennedy, The particle swarm-explosion, stability, and convergence in a multidimensional complex space, IEEE Transactions on Evolutionary Computation, 6(2), 2002, 58-73.

[21] A.I. El-Gallad, M.E. El-Hawary, A.A. Sallam, \& A. Kalas, Enhancing the particle swarm optimizer via proper parameters selection, Canadian Conference on Electrical and Computer Engineering, 2002, 792-797.

[22] D. Bratton and T. Blackwell, A Simplified Recombinant PSO, Journal of Artificial Evolution and Applications, 2008.

[23] Y. Shi \& R. Eberhart, A modified particle swarm optimizer, Proc. IEEE World Congr. Computational Intelligence Evolutionary Computation, 1998, 69-73.

[24] Y. Shi \& R. Eberhart, Empirical study of particle swarm optimization, Proc. Congr. Evolutionary Computation (CEC 99), vol. 3, 1999, 1945-1950.

[25] F. van den Bergh \& A.P. Engelbrecht, Effects of swarm size on cooperative particle swarm optimizers, Proc. Genetic and Evolutionary Computation Conference 2001, San Francisco, USA, 2001.

[26] G. Evers, An Automatic Regrouping Mechanism to Deal with Stagnation in Particle Swarm Optimization, Master's thesis The University of Texas - Pan American, Department of Electrical Engineering, USA, 2009.

[27] Ansoft High Frequency Structure Simulator (HFSS), ver. 12.1, Ansoft Corporation, Pittsburgh, PA, 2009.

[28] C. Karul, S. Soyupak, and C. Yurteri, Neural network models as a management tool in lakes, in journal of Hydrobiologia 408/409, pp. 139-144, 1999. 